Cerebrovasc Dis 2007;23:315-317

DOI: $10.1159 / 000098446$

\section{Spontaneous Intracranial Hypotension Is Associated with a Risk of Venous Sinus Thrombosis and Subdural Hematoma}

Hiroshi Kataoka, Emi Tanizawa, Satoshi Ueno

Department of Neurology, Nara Medical University, Kashihara, Nara, Japan

\section{Introduction}

Spontaneous intracranial hypotension (SIH) is determined by cerebrospinal fluid pressure of $6 \mathrm{~cm} \mathrm{H}_{2} \mathrm{O}$ or less in patients with no history of lumbar puncture. The holocranial headache of $\mathrm{SIH}$ is elicited or exacerbated in the upright position. Less commonly, hearing disturbance, diplopia and neck pain occur. SIH is sometimes associated with subdural collections of fluid; however, large subdural hematomas requiring urgent neurosurgical drainage are very rare. Although cerebral venous sinus thrombosis (VST) has a wide variety of risk factors and causes, the association with SIH remains unclear. We describe a patient with SIH who had VST and large subdural hematomas, both rare complications.

\section{Case Report}

On November 30, 2005, a 36-year-old man presented with bilateral auditory distortion, which rapidly disappeared when he was lying flat. The patient had no history of previous illness, major trauma or dural puncture. By December 1, occipital headache and neck pain with auditory distortion occurred while sitting. Two days later, holocranial headache with nausea and auditory distortion developed while sitting or standing. These symptoms resolved when he laid down. On December 23, he had difficulty in moving the left extremities with increasing headache in the upright position for about $15 \mathrm{~min}$. These symptoms gradually disappeared within half an hour of recumbency. Because of intense postural headache, he was admitted to our hospital on December 27. On admission, the results of fundus, neurological and general examinations were normal, except for headache. An enhanced CT scan showed an empty delta sign, typical of superior sagittal sinus (SSS) thrombosis (fig. 1A). Brain magnetic resonance imaging (MRI) showed downward displacement of the cerebellar tonsil (fig. 1B). There was no parenchymal lesion. On gadolinium-enhanced $\mathrm{T}_{1}$-weighted images, there was diffuse pachymeningeal enhancement (fig. 1C). Magnetic resonance venography (MRV) confirmed markedly reduced flow in the SSS (fig. 1D). At lumbar puncture, the CSF opening pressure was $4 \mathrm{~cm} \mathrm{H}_{2} \mathrm{O}$ while lying down. The CSF contained 14 lymphocytes and $143 \mathrm{mg} / \mathrm{dl}$ of protein. Complementary screening studies for infectious, immunologic, hematologic, neoplastic and systemic disorders, including tests for protein C, antiphospholipid antibodies and anticardiolipin antibodies, showed no apparent cause of VST. There was no family or personal history of clotting disorders or use of oral contraceptives. The patient was given a diagnosis of VST with SIH and received intravenous heparin, followed by oral anticoagulants. On January 3, 2006, he noted left hemiparesis with headache and auditory distortion in the upright position. After half an hour in a supine position, all manifestations disappeared. On January 24, radionuclide cisternography demonstrated a CSF leak at the cervicothoracic junction (fig. 1E). The postural headache worsened from 1 day after cisternography onward and became permanent. Repeat cranial MRI on February 1 showed mild subdural hematomas in addition to VST. Anticoagulant therapy was stopped. On February 3, he was treated with an epidural blood patch. Subsequently, the headache decreased in intensity. However, on February 15, he presented with severe headache in both the upright and supine positions, with vomiting and meningeal stiffness. Cranial CT showed bilateral increases in the hematomas in the subdural space with mass effect (fig. 1F). The subdural hematomas were urgently drained through bilateral burr holes. Repeated cranial enhanced MRI on February 25 showed a reduction in the subdural hematomas and meningeal enhancement; however, it revealed no recanalization of the SSS. The patient, who was receiving no anticoagulant medication, had relief of headache and was discharged on March 11. Follow-up MRV on April $26 \mathrm{dem}-$ onstrated dramatic recanalization of the SSS (fig. 1G). Cranial MRI showed that the cerebellar tonsil had risen; there was no meningeal enhancement (fig. $1 \mathrm{H}, \mathrm{I}$ ).

\section{Discussion}

An association of SIH with VST is extremely rare. To our knowledge, only 4 cases of SIH with VST have been reported, including 3 with other risk factors for thrombosis such as congenital thrombophilia or steroid therapy [1-3]. VST can develop after spinal tap, myelography or spinal anesthesia; however, most cases have also had other potential risk factors for hypercoagulable states [4]. We found no probable cause of either VST or hypercoagulable state. Our patient had dramatic recanalization of the venous sinus in response to an epidural blood patch, without receiving continuous anticoagulant therapy. Furthermore, the intermittent and postural headache preceded difficulty in moving the left extremities at the initial course of SIH. We believe that intracranial hypotension (IH) directly caused VST. With an intact skull, the overall volume of the brain, CSF and intracranial blood

\section{KARGER}

Fax +41613061234 E-Mail karger@karger.ch www.karger.com
(2) 2007 S. Karger AG, Basel

$1015-9770 / 07 / 0234-0315 \$ 23.50 / 0$

Accessible online at:

www.karger.com/ced 

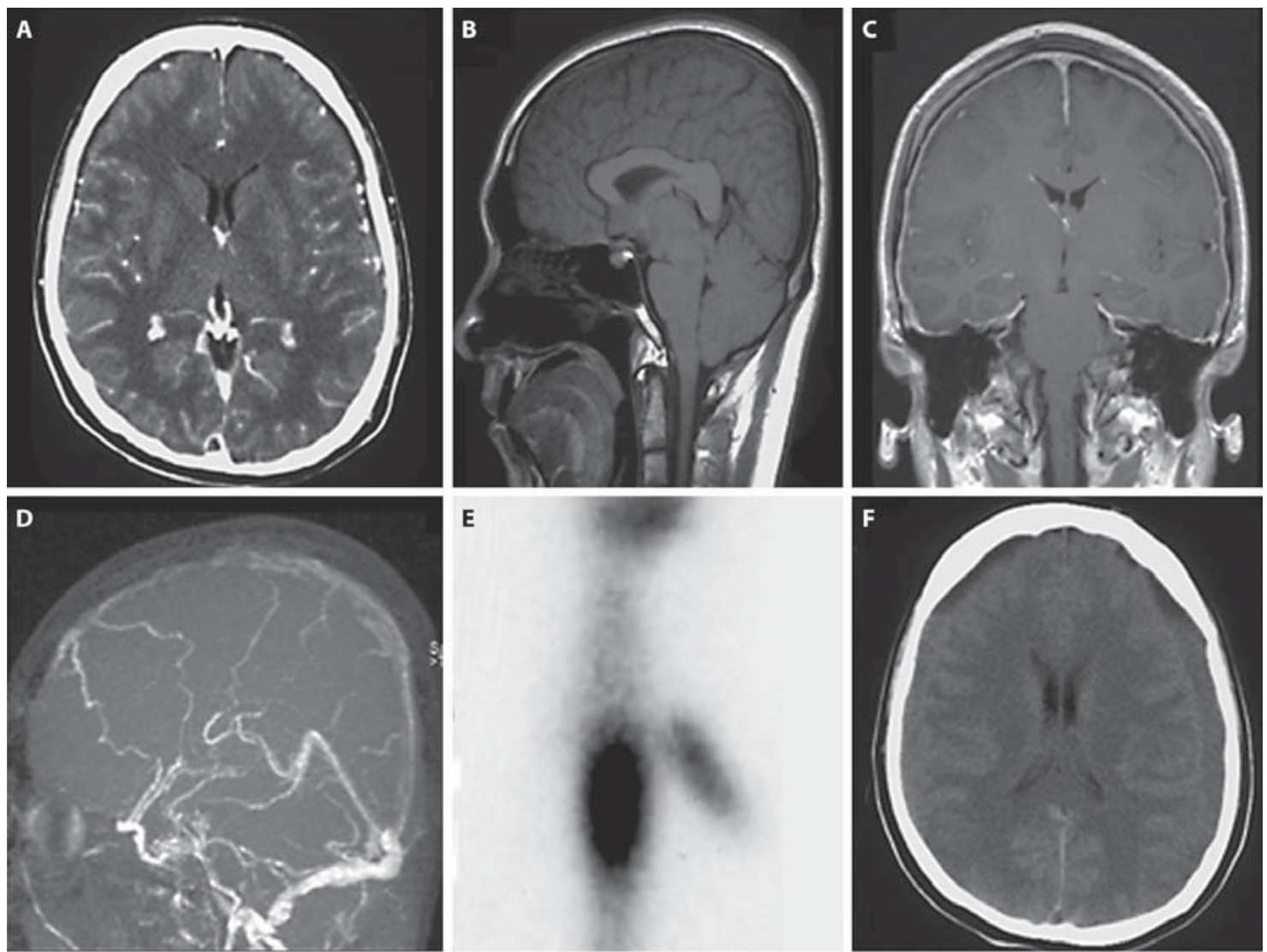

E
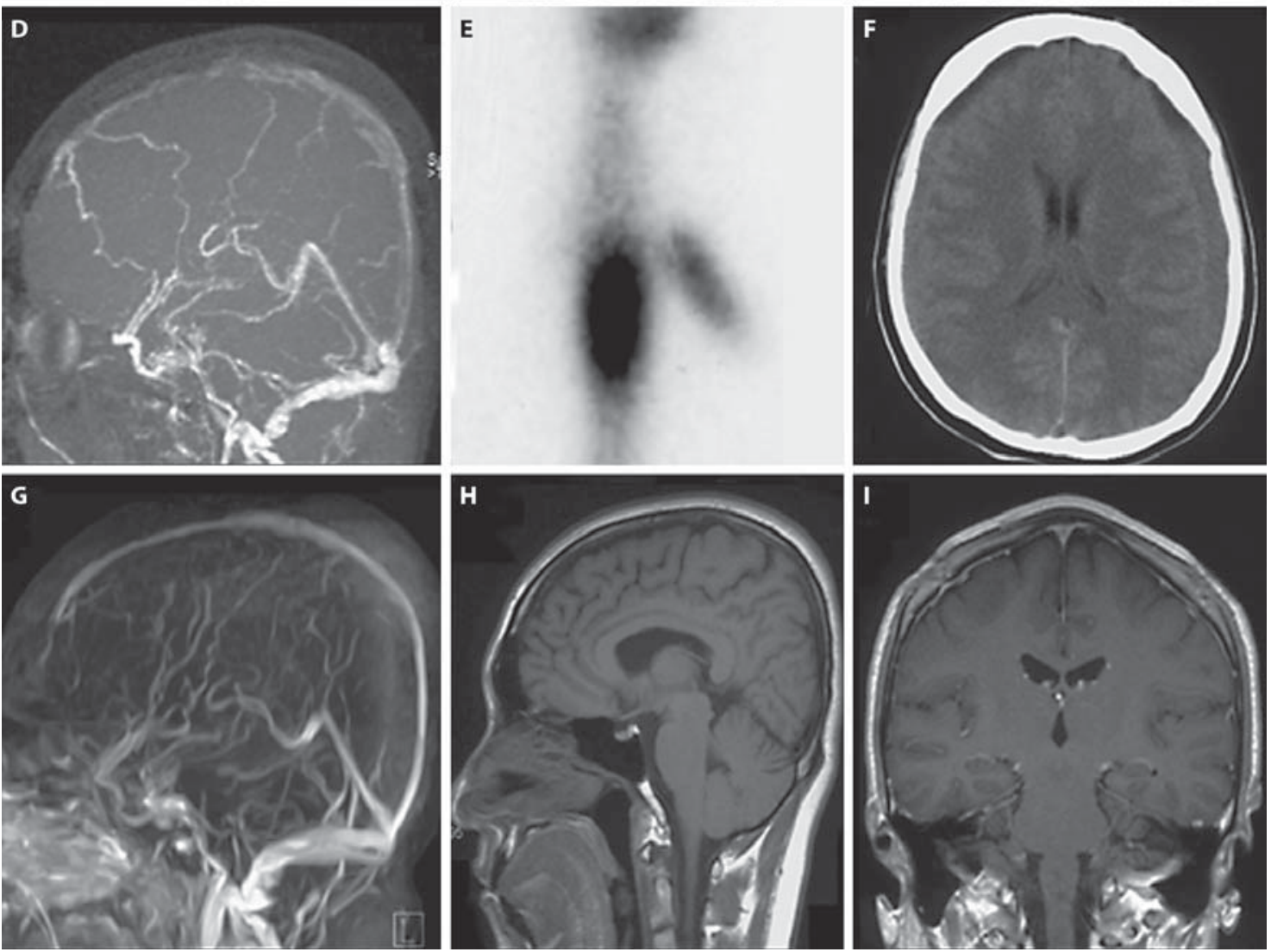

Fig. 1. Cranial enhanced CT showed an empty delta sign. A $\mathrm{T}_{1}$-weighted MRI showed descending cerebellar tonsil. B Enhanced coronal $\mathrm{T}_{1}$-weighted MRI with the use of gadolinium diethylenetriamine penta-acetic acid (Gd-DTPA) demonstrated symmetric meningeal enhancement. C MRV showed markedly decreased flow within the superior sagittal sinus. D Radionuclide cisternography in the anterior projection of the head and upper spine. Diffusion of tracer can be seen outlining the spinal roots near the cervicothoracic junction. E Cranial CT demonstrated increasing subdural hematomas with mass effect. F MRV demonstrated recanalization of the superior sagittal sinus. G Follow-up MRI showed that the cerebellar tonsil had risen on $\mathrm{T}_{1}$-weighted image $(\mathbf{H})$, with no meningeal enhancement on Gd-DTPA-enhanced image (I). 
remains constant [5]. An increase in the volume of 1 of these components thus produces a decrease in the remaining 2 volumes. Reductions in CSF volume and pressure require a compensatory increase in intracranial blood volume, primarily in the venous system, since the brain volume changes only slightly [5]. In $\mathrm{SIH}$, CSF leakage increases the dural venous blood volume and slows the blood flow, leading to stasis of the dural venous system. Such stasis is a potential cause of hypercoagulable states. Our data suggest that IH may have a risk of VST. Moreover, the patient presented with transient hemiparesis and headache in the upright position. Low CSF pressure aggravated by an upright position may increase venous vasodilatation, altering the venous circulation and leading to hemiparesis. This phenomenon also supports the relation between SIH and VST.

Subdural hematoma in our patient was associated with the change in headache pattern. Headache became persistent not only in the upright but also in the supine position. Although the incidence of subdural collections associated with SIH is $10 \%$ [6], large subdural hematomas requiring urgent neurosurgical drainage are rare. Downward displacement of the brain due to low CSF pressure may produce tears in bridging veins located in the layer of dural border cells, causing these veins to rupture, leading to subdural hematoma [7]. Headache due to low CSF pressure is typically intermittent and postural, characterized by intense holocranial headache while sitting or standing, which is relieved by lying flat [4]. Downward sagging of the brain produces headache by applying traction to pain-sensitive structures, in particular cranial nerve $\mathrm{V}$ [7]. Increased intracranial pressure usually leads to persistent headache, often worse in the supine position [4]. In IH syndrome, this change in the headache pattern is a crucial symptom of subdural hematoma.

SIH shares common risk factors with VST and subdural hematoma. The clinician should be aware of potential risks.

\section{References}

1 Sopelana D, Marcos A, Arroyo R, Gutierrez E, Cuenca R, Vazquez AV, Gonzalez JL, Egido JA: May intracranial hypotension be a cause of venous sinus thrombosis? Eur Neurol 2004;51:113-115.

2 Berroir S, Grabli D, Heran F, Bakouche P, Bousser MG: Cerebral sinus venous thrombosis in two patients with spontaneous intracranial hypotension. Cerebrovasc Dis 2004;17:9-12.

3 Flemming KD, Link MJ: Spontaneous CSF leak complicated by venous thrombosis and dural arteriovenous fistula. Cephalalgia 2005;25:751753

4 Aidi S, Chaunu MP, Biousse V, Bousser MG: Changing pattern of headache pointing to cerebral venous thrombosis after lumbar puncture and intravenous high-dose corticosteroids. Headache 1999;39:559564.

5 Mokri B: The Monro-Kellie hypothesis: applications in CSF volume depletion. Neurology 2001 26;56:1746-1748.

6 Renowden SA, Gregory R, Hyman N, Hilton-Jones D: Spontaneous intracranial hypotension. J Neurol Neurosurg Psychiatry 1995;59:511515.

7 Pannullo SC, Reich JB, Krol G, Deck MD, Posner JB: MRI changes in intracranial hypotension. Neurology 1993;43:919-926.

Dr. H. Kataoka

Department of Neurology

Nara Medical University, 840 Shijo-cho

Kashihara, Nara 634-8522 (Japan)

Tel. +81 74429 8860, Fax +81 744246065

E-Mailhk55@naramed-u.ac.jp 